\title{
ARTICLE
}

Clinical Research

\section{Molecular medicine tumor board: whole-genome sequencing to inform on personalized medicine for a man with advanced prostate cancer}

\author{
Andrew J. Armstrong $\mathbb{1}^{1} \cdot$ Xiaotong $\mathrm{Li}^{2} \cdot$ Matthew Tucker $^{3} \cdot$ Shantao $\mathrm{Li}^{2} \cdot$ Xinmeng Jasmine $\mathrm{Mu}^{4}$. \\ Kenneth Wha $\mathrm{Eng}^{5} \cdot$ Andrea Sboner ${ }^{6} \cdot$ Mark Rubin $\mathbb{1}^{6,7,8} \cdot$ Mark Gerstein $\mathbb{1}^{2,9}$
}

Received: 9 April 2020 / Revised: 14 December 2020 / Accepted: 15 January 2021 / Published online: 10 February 2021

(c) The Author(s) 2021. This article is published with open access

\begin{abstract}
Purpose Molecular profiling of cancer is increasingly common as part of routine care in oncology, and germline and somatic profiling may provide insights and actionable targets for men with metastatic prostate cancer. However, all reported cases are of deidentified individuals without full medical and genomic data available in the public domain.

Patient and methods We present a case of whole-genome tumor and germline sequencing in a patient with advanced prostate cancer, who has agreed to make his genomic and clinical data publicly available.

Results We describe an 84-year-old Caucasian male with a Gleason 10 oligometastastic hormone-sensitive prostate cancer. Whole-genome sequencing provided insights into his tumor's underlying mutational processes and the development of an $S P O P$ mutation. It also revealed an androgen-receptor dependency of his cancer which was reflected in his durable response to radiation and hormonal therapy. Potentially actionable genomic lesions in the tumor were identified through a personalized medicine approach for potential future therapy, but at the moment, he remains in remission, illustrating the hormonal sensitivity of his $S P O P$-driven prostate cancer. We also placed this patient in the context of a large prostate-cancer cohort from the PCAWG (Pan-cancer Analysis of Whole Genomes) group. In this comparison, the patient's cancer appears typical in terms of the number and type of somatic mutations, but it has a somewhat larger contribution from the mutational process associated with aging.

Conclusion We combined the expertise of medical oncology and genomics approaches to develop a molecular tumor board to integrate the care and study of this patient, who continues to have an outstanding response to his combined modality treatment. This identifiable case potentially helps overcome barriers to clinical and genomic data sharing.
\end{abstract}

These authors contributed equally: Andrew J. Armstrong and Xiaotong Li

Supplementary information The online version contains supplementary material available at https://doi.org/10.1038/s41391021-00324-5.

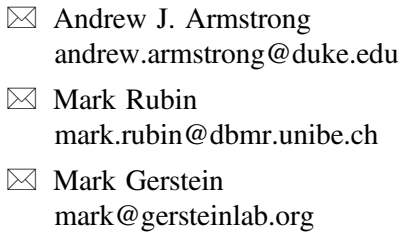

Extended author information available on the last page of the article

\section{Introduction}

Over the last 20 years, the genomic heterogeneity of prostate cancer (PC) has become increasingly recognized across the spectrum of localized and metastatic disease [1-5]. Emerging data support that PC is driven by a number of genetic variants, including specific gene fusions, gains and losses of chromosomal regions, point mutations, and variations in epigenetic signatures (e.g., histone methylation). These subgroups may provide additional prognostic stratification. For example, with combined genomic/epigenomic subgrouping of PC data from the Cancer Genome Atlas, nearly three-fourths of all PC cases could be categorized into one of four gene fusion groups (ERG, ETV1, ETV4, and FLII) or three gene mutation groups (SPOP, FOXA1, and IDH1) [4]. Moreover, genomic subtypes including luminal and basal classifications can be differentiated based on clinical outcomes [6]. 
In the clinic, men with metastatic PC frequently harbor germline or somatic defects in traditional DNA repair genes such as BRCA1, BRCA2, ATM, and FANCD2, with 20\% of cases harboring such deficiencies [2, 4, 7]. Therefore, national guidelines now recommend germline testing for all high-risk and metastatic patients, and for those with a family history suggestive of cancer. Such alterations in DNA repair enzymes may impact familial risk and genetic counseling as well as opportunities for therapy, such as recently FDA-approved poly ADP-ribose polymerase inhibitors for tumors with known DNA repair defects or pembrolizumab for men with microsatellite-high and/or mismatch repair-deficient tumors [8, 9].

Many centers are increasingly using targeted nextgeneration sequencing panels which include many of the most common mutations including DNA repair and mismatch repair enzymes as well as somatic biallelic $C D K 12$ inactivation, a newly described immunogenic subset of prostate cancer [10-12]. However, with falling price of sequencing and the possibility of identifying novel genomic events, whole-genome approaches may provide additional clinical benefits beyond targeted sequencing.

Here, we provide a case report describing the diagnosis and treatment of a man with primary M1 oligometastatic PC who volunteered to release his entire protected health information, including the results of whole-genome sequencing (WGS) of his germline and both whole-exome sequencing (WES) and WGS of his PC. Through a multiinstitutional clinical-molecular tumor board, we integrated clinical, somatic, and germline genomic sequencing data, and used bioinformatics to put his tumor in the context of (1) existing knowledge of PC biology, (2) his outstanding response to combined radiation and hormonal therapy, and (3) future clinical management recommendations. This case study demonstrates the utility of translational genomics in cancer precision medicine.

\section{Patient and methods}

After diagnosis, the patient provided informed consent and underwent WES and WGS of both tumor tissue (tumor content $=66.9 \%$ ) and normal blood samples. For WES, the tumor was sequenced to an average coverage of 104x using an Agilent HaloPlex, covering 21,522 genes. Sequencing was performed using the Illumina HiSeq 2500 platform. Sequencing reads were aligned to the GRC37/hg19 reference and processed according to the WES Test for CancerExaCT1-pipeline v0.9 with a capture efficiency of $90.29 \%$ [13]. For WGS, the average sequence depth was $\sim 120 \mathrm{X}$ for the subject and $\sim 60 \mathrm{X}$ for the matched normal. We took the intersection of somatic variants call sets generated by MuTect [14] and Strelka [15]. Using all sequencing data, we analyzed the subject's germline and somatic mutations in comparison with a cohort of WGS PC cases [16]. We processed the germline WGS data using a standard pipeline following GATK [17] best practices. The patient also provided reports from a commercial Hereditary Cancer Risk Test (color assay on saliva; $n=30$ genes), and a HIPAA waiver for disclosure of his personal and genomic data. Computer code used to generate the results in this study is available from the corresponding author on reasonable request.

\section{Results}

At the time of initial presentation in 2015, the patient was 79 years old and was being followed closely by his primary care provider for increasing lower urinary tract symptoms, including increased frequency, urgency, nocturia, and weakened stream. The patient is of Northern European ancestry and had a positive family history for $\mathrm{PC}$ in a cousin (non-lethal, age 68) and paternal uncle, and multiple family members with lung cancer (daughter, sister, mother, son). He was a former smoker (10 pack-years) and retired investment banker and economist without other significant exposures. He had been followed with yearly prostate specific antigen (PSA) levels since age 50 in the normal range $(<4.0 \mathrm{ng} / \mathrm{mL})$ and with normal digital rectal examinations, but repeat PSA testing demonstrated a dramatic increase to $13.08 \mathrm{ng} / \mathrm{mL}$ on $1 / 19 / 2015$ with digital rectal exam notable for an indurated prostate with bilateral nodularity measuring $\sim 50 \mathrm{cc}$ volume (cT2 stage).

The patient underwent transrectal ultrasound-guided biopsy on 2/10/2015, revealing a bilateral Gleason $5+$ $5=10$ adenocarcinoma (Grade Group 5) with 12/12 cores positive for high-volume, high-risk disease, including regions of Gleason $5+3=8$ and $4+5=9$ adenocarcinoma, without small-cell features. Perineural invasion was noted diffusely. Staging bone scintigraphy using ${ }^{99 \mathrm{~m}} \mathrm{Tc}$ with methylene diphosphonate showed a focus of increased radiotracer activity in the left ischium, which correlated with computerized tomography (CT) imaging, consistent with osseous metastatic disease (Fig. 1). Computerized tomography imaging showed no evidence of lymphadenopathy or visceral metastatic disease. As such, the patient was staged as T2bNXM1 PC and began androgen deprivation therapy (ADT) on 2/12/2015 with once-daily bicaluatmide $50 \mathrm{mg}$ for 30 days along with a triptorelin pamoate $22.5 \mathrm{mg}$ depot injection.

On $5 / 11 / 2015$, the patient's repeat PSA was down to $0.09 \mathrm{ng} / \mathrm{mL}$ (Fig. 1A) and repeat bone scintigraphy showed decreased radiotracer activity in the left ischium and no new evidence of metastatic disease (Fig. 1B-D). His PSA increased slightly to $0.11 \mathrm{ng} / \mathrm{mL}$ on $7 / 21 / 2015$ and he 
A.

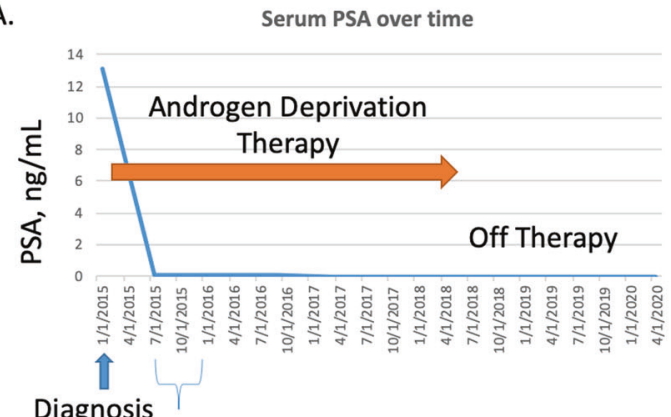
Radiation
(IMRT)
B.

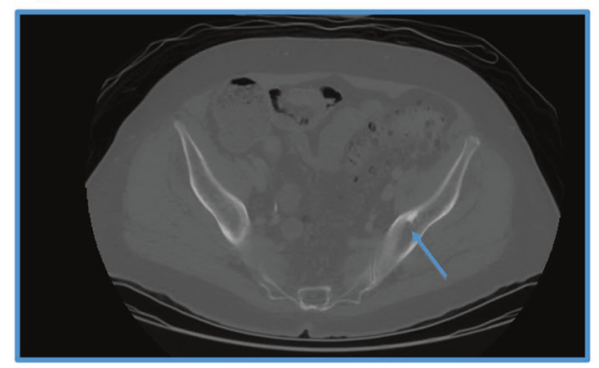

Post-Treatment Bone Scan

D.

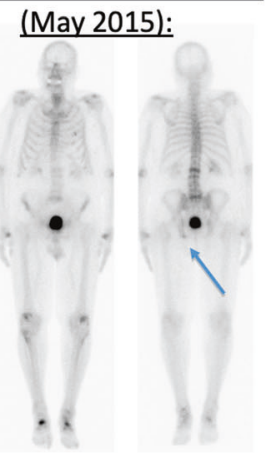

E.

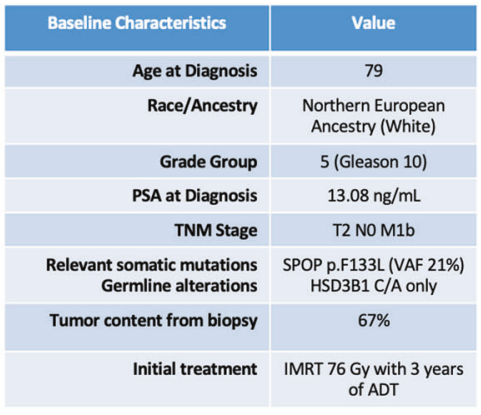

Fig. 1 Summary of the patient case and outcomes. A Response of serum PSA to initial therapy with IMRT and 3 years of ADT, with ongoing response off therapy now for 2 years. B Staging CT at diagnosis demonstrating a left pelvic/ischia osteoblastic metastasis, confirmed on $\mathbf{C}$ bone scan at baseline/diagnosis in 2015 and $\mathbf{D}$ subsequent to therapy 3 months after treatment initiation, showing a favorable treatment effect. E Summary table of our patient's case report. subsequently underwent transrectal ultrasound-guided fiducial marker placement, along with 38 fractions of intensity-modulated radiation therapy (IMRT) to his prostate and seminal vesicles. From 8/18/2015 to 10/2/2015, he received 15 fractions of IMRT boost to his prostate for a total of 7600 centigray. His ADT was switched to leuprolide acetate $45 \mathrm{mg}$ intramuscularly and was continued every six months. No pelvic or metastatic site radiation was performed. One year after diagnosis $(1 / 25 / 2016)$, the patient's PSA levels were $0.05 \mathrm{ng} / \mathrm{mL}$. From September 2016 through September 2018 he was maintained on ADT and his PSA remained undetectable. His ADT was held in October 2018 and his PSA has remained 0.01-0.02 through his most recent checkup on 4/1/2020 (Fig. 1). Overall, the patient has tolerated ADT well with minimal adverse effects that include weight gain, intermittent hot flashes, and decreased libido. He began intermittent ADT in 2018 and presently remains off therapy through November 2020 and free of detectable disease at the age of 83 with a PSA of 0.01 . Adverse effects from radiation were also minimal and consisted primarily of intermittent diarrhea that resolved. Adverse effects from hormonal therapy have included weight gain, mild to moderate fatigue, muscle loss, and mild hot flashes. The patient's summary clinical data is provided in Fig. 1E.

Throughout the course of treatment, the patient has maintained a healthy lifestyle with regular exercise, emphasizing restorative yoga, and a predominately vegan diet. He does not drink alcohol and has a remote five-year history of cigarette smoking, having quit in 1964 . He studied system theory, mathematics, and predictive algorithms in economics and epistemology and has been very involved in his treatment decisions and genomic analyses.

After providing informed consent, the patient underwent WES and WGS somatic and germline analysis at Weill Cornell Medical College as part of a Precision Medicine research study, in conjunction with clinical care at the Duke Cancer Center and Duke Cardiology. Integrated bioinformatics analysis was performed at Yale University.

The patient first underwent standard-of-care germline testing using a 30-gene-panel assay for hereditary DNA repair defects and other pathogenic clinical mutations associated with hereditary PC or unfavorable clinical outcomes [7]. Using a commercial Hereditary Cancer Risk Test on 12/31/16, no mutations were identified in 31 DNA homologous or mismatch repair genes, including $B R C A 1 / 2$, ATM, POLD1/POLE, RAD51D, TP53, NBN, PALB2, PMS2, MLH1, MSH2, MSH6, MYTYH, and EpCAM. In addition, immunohistochemistry analysis of his prostate biopsy did not reveal loss of $M L H 1, M S H 2, M S H 6$, or $P M S 2$, and his tumor was found to be microsatellite stable.

On $6 / 21 / 2016$, the patient's prostate biopsies were subjected to next-generation WES and WGS with a matched normal control at Weill Cornell Medical College. Germline 
Fig. 2 Enrichment of GWAS risk alleles. A The number of heterogenous states of risk alleles (i.e., carry one risk allele) in an individual. B The number of homogenous states of GWAS risk alleles (i.e., carry two risk alleles) in an individual. In both cases, our subject carries slightly higher risk alleles (z-score: +0.36 and +1.0 respectively).

Fig. 3 Somatic mutatioal signatures and tumor heterogeneity. A A dotchart showing the fractions of activities of signatures in the subject (blue) and average PCAWG individuals (red). The subject has higher signature 5 . B The subject (red) when compared to the PCAWG individuals (gray), has slightly higher mutation load (y-axis) and higher MATH score ( $x$-axis).
A)

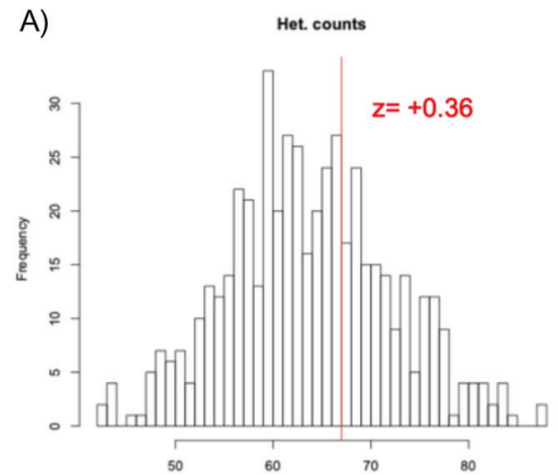

A)

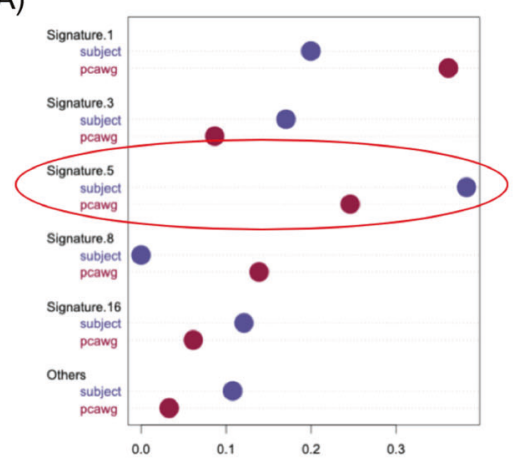

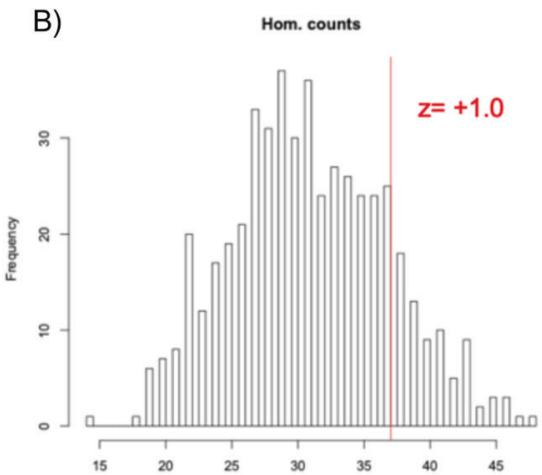

B)

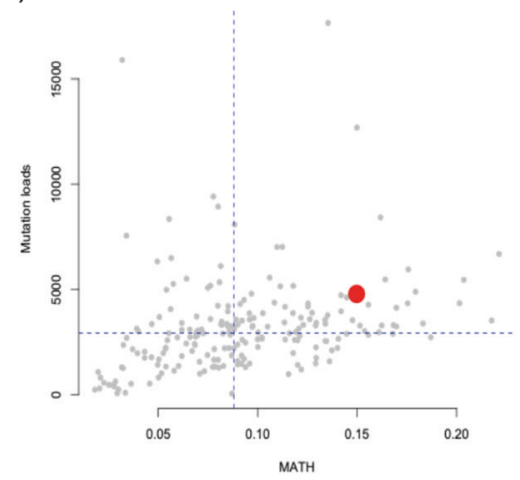

WGS data further confirmed that the subject did not carry any significant germline mutations in DNA repair genes. In a panel of genome-wide association study (GWAS) risk alleles $(N=428)$, his genome demonstrated insignificant enrichment for risk alleles when compared to 503 genomes of European ancestry (EUR) from the 1000 Genomes Project [18], carrying 67 heterozygous risk alleles (average in EUR: 64; $\mathrm{z}=+0.36$ ) (Fig. 2A) and 37 homozygous risk alleles (average in EUR: $31 ; \mathrm{z}=1.0$ ) (Fig. 2B). Additionally, the subject does not carry the HOXB13 G84E variant, which is associated with a significantly higher risk of hereditary PC [19].

Studies have shown that a germline missense variation (rs1047303) in HSD3B1 is predictive for ADT failure [2022], especially in individuals with homozygous risk alleles $(\mathrm{C} / \mathrm{C})$. WGS revealed that our patient is heterozygous (C/A) for this single-nucleotide polymorphism (SNP), suggesting a prolonged and more robust response to ADT. Somatic loss of heterozygosity of this variant has been reported to be a significant event in PC; however, we did not find evidence for this in his tumor sample (frequency of allele $=0.508$ in germline, 0.659 in somatic). Recent evidence suggests that individuals with rs1047303 might metabolize abiraterone differently and this may be associated with androgen receptor (AR) agonism; thus, the clinical activity of abiraterone acetate is uncertain should he continue with ADT, and alternatives such as enzalutamide may be considered [23].
Using WGS, we found that the subject carries 4780 SNPs (z-score $=+0.47, \log$-transformed) and 332 insertions/deletions. We further stratified the SNPs into 58 coding mutations (z-score after normalized by mutation load $=-0.73)$ and 66 high-functional-impact noncoding mutations (FunSeq [24] score >1.5; z-score after normalized by mutation load $=+1.11$ ). The Variants Effect Predictor [25] found 0 high-impact and 44 moderate-impact mutations (z-score after normalizing by mutation load $=$ -1.02 and +0.53 , respectively).

Using sigLASSO [26] and 30 COSMIC [27] signatures, we next examined somatic mutational patterns. The subject showed a large amount of activity from signature 5 (Fig. 3A). This signature has been demonstrated to be simply associated with age [28], consistent with the subject's advanced age (81 versus 58 in the Pan-Cancer Analysis of Whole Genomes (PCAWG) cohort [29]). The patient showed a low percentage of signature 1, with characteristic spikes associated with $\mathrm{C}>\mathrm{T}$ mutations in CpGs, indicating reduced genome methylation. Indeed, when comparing the percentage of $\mathrm{C}>\mathrm{T}$ mutations in CpGs, our patient's tumor ranks in the lower quartile within the published cohort (rank: 31/200, z-score $=-1.06$ ). We did not find other significant somatic mutational processes such as homologous repair deficiency or ApoBEC. We also calculated the mutant-allele tumor heterogeneity (MATH) score [30] using somatic mutations. In comparison with the 


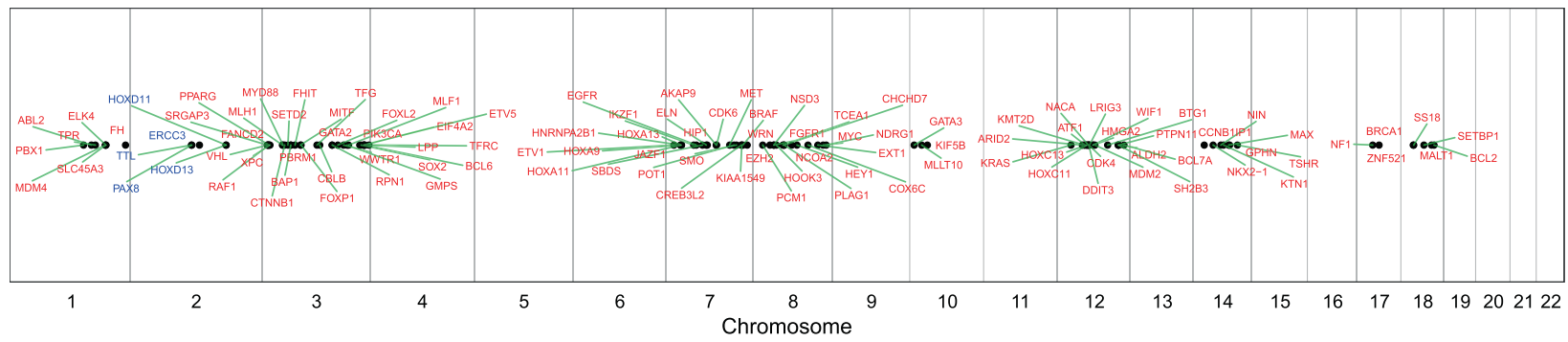

Fig. 4 Cancer genes with copy number alterations. Cancer genes reporting genomic alterations identified from the subject were listed based on their locations on chromosomes. Genes in red had copy number gain events. Genes in blue reported copy number loss events.
PCAWG cohort, the subject showed a slightly higher score, which could indicate greater tumor heterogeneity. However, we suspect that this result is largely due to the patient's higher mutation load (Fig. 3B).

In the targeted gene panel, we sequenced 59 clinically relevant genes linked to FDA-approved therapies and did not identify any actionable alterations. Additionally, we evaluated 575 known cancer genes using the WES results, and identified 20 alterations (Fig. 4, Supplementary Table 1). Finally, we identified 18 genes with point mutations or insertions/deletions with unknown clinical significance (Supplementary Table 2), including a missense mutation in the speckle-type pox virus and zinc finger protein $(S P O P)$ gene (p.F133L) with a tumor variant allele frequency of $20.6 \%$. Notably, this amino-acid change is highly recurrent in a large panel of PC samples [31] (3.6\%, $36 / 1,013)$. The patient does not have a germline or somatic variant of rs1376350, a SNP at 7p14.3 that can associate with the SPOP mutation [32].

Although we did not identify additional common PCdriver mutations (e.g., AR, RB1, PTEN, MYC, or TP53), we did identify copy number alterations (Fig. 4, Supplementary Table 1). For example, we found a broad copy gain (likely trisomy) of chromosome 7 and 8 encompassing $M E T$, $E Z H 2, E G F R$, and $M Y C$; a broad copy gain of chromosome $3 \mathrm{q}$ encompassing the $S O X 2$ and PIK3CA loci; gain of $3 \mathrm{p}$ and the FGFR1 locus; and copy losses at $2 \mathrm{q}$ encompassing the PAX8 and ERCC3 loci as well as the HOXD11 and $H O X D 13$ loci. We did not find evidence of CHDl loss, which often co-occurs with SPOP mutations in PC. We observed deletions disrupting the estrogen receptor ESRI on chromosome 6. Multiple structural variant discovery tools have reported interchromosomal translocation and inversion events in ESR1, however the clinical significance is unknown.

Finally, we investigated the mutational burden on the entire genome with regards to epigenetics. To estimate the mutational burden, we tabulated the number of mutations observed in functional genomic regions as defined by epigenetic markers. We used ChromHMM [33] to segment the genome into 15 basic epigenetic states (e.g., enhancer, promoter) based on five histone modification signals. This is the first ChromHMM segmentation reported for primary prostate tissue. Next, we tallied the number of mutations of the subject and 199 PCAWG samples in each state. We found no significant differences between the subject and the PCAWG cohort in mutational burden (absolute $\mathrm{z}$-scores $<2$ for all 15 states).

\section{Discussion}

\section{Significance of SPOP mutations}

Here, we provide the first identifiable molecular tumor board case report of a patient with advanced PC. Our analysis includes comprehensive germline and somatic tumor whole-genome profiling as well as consideration of clinical outcomes. We found that this patient harbored oligometastatic hormone-sensitive PC driven by an age-related mutational process and higher-risk germline SNPs, leading to a pathogenic SPOP mutation. The patient is presently responding well to intermittent $\mathrm{ADT}$ and is expected to respond well based on the AR dependence of SPOP-mutant $\mathrm{PC}$ and his heterozygous HSD3Bl gene. Beyond the genomic findings, this case report is unique as it details the patient's goals, identity, medical history, preferences, and clinical outcomes.

While we did not identify any currently actionable mutations, the SPOP mutation has the potential to be actionable in the near future. SPOP is an E3-ubiquitin ligase with several substrates including the AR, and mutations disrupt proteosomal degradation, accumulation, and AR activity [34, 35]. Non-synonymous mutations in SPOP such as the F133L mutation in our patient are found in about $10 \%$ of PC cases [36]. The F133L alteration suggests an AR-dependent tumor, consistent with the patient's prolonged clinical response to standard ADT. This alteration is enriched in localized-PC and de-enriched in mCRPC datasets [37, 38], suggesting that SPOP mutations select for hormonally responsive tumors with excellent outcomes. 
Studies have shown that the SPOP enzyme is directly involved in homologous DNA repair. In vitro models knocking out $S P O P$ demonstrate suboptimal DNA repair as well as increased sensitivity to ionizing radiation [35, 39]. Furthermore, studies using both mouse- and human-derived PC cell lines have shown that mutant SPOP (including $S P O P-\mathrm{F} 133 \mathrm{~V})$ in concert with CHD1 loss exhibits increased double-strand break repair and sensitivity to DNA-damaging agents such as poly ADP-ribose polymerase inhibitors [40]. Recent multicenter data from men with mHSPC treated with ADT suggest that SPOP mutations confer a favorable long-term prognosis on progression free and overall survival, which aligns with the current excellent outcome of our current patient [41].

\section{Analyses of the whole genome}

In addition to $S P O P$, other cancer driver genes are altered in the subject's tumor genome (Supplementary Table 3). Previous studies have shown that a germline missense variation (rs1047303) in the HSD3B1 gene is predictive for ADT failure [20-22], especially in individuals with homozygous risk alleles $(\mathrm{C} / \mathrm{C})$. Based on the patient's heterozygous HSD3B1 SNP, his response and metabolism of abiraterone with resultant AR agonism might be of some concern; thus, enzalutamide, apalutamide, or darolutamide may be preferred over abiraterone acetate should the disease progress [23].

MET activation promotes tumor growth and metastasis. Therefore, inhibiting MET has been attractive therapeutically in several cancer types [42]. In PC, this therapy did not extend survival in Phase 3 clinical trials of unselected men. However, this trial was conducted in men heavily pretreated with $\mathrm{mCRPC}$ who harbored tumors that were likely highly heterogeneous with a high burden of metastases [43]. Cabozantinib may have greater activity in $M E T$-amplified tumors, and its utility is unknown in earlier lines of therapy. Other oncologic processes of PC are being actively investigated. Genes regulating the epigenome and metabolic pathways have shown tremendous therapeutic potential [44]. In addition, although PC is considered an immunologically 'cold' tumor, active research and clinical trials aim to induce an immune response to PC, thereby turning 'cold' tumors 'hot'. These advances present many potential treatment opportunities for PC patients.

With rich mutational information from WGS, we also explored the mutational landscape of the sample. We examined noncoding regions and explored mutational signatures, regulatory region mutational burden, and tumor heterogeneity. We determined that the underlying mutational process was most likely related to an age-related signature (signature 5), rather than a hereditary DNA repair deficiency or carcinogenic/exposure signature [28].

\section{Publicly available data}

Our subject agreed to release all of his genomic sequencing data for public use, which has been deposited into the European Genome-Phenome Archive (EGAS00001004648). As described, this case is an elderly Caucasian man with highrisk bone oligometastatic $\mathrm{PC}$, harboring a classic SPOP mutation, who has been well managed by IMRT and ADT and is presently enjoying a prolonged treatment-free interval. Our patient underwent a range of personal genomics tests, including direct-to-consumer commercial genetic tests as well as standard medical diagnostic WES and WGS on both germline and somatic cancer tissues over several years. These results form a rich genomic dataset of various quality, coverage, and resolution, and provide a unique opportunity to study how genomic sequencing operates in the real world. Importantly, we processed and provided rich annotations on the sequencing data, such as mutational patterns and signatures and epigenomic impacts, and compared his mutational profiles with 199 WGS PC samples from the PCAWG and other public datasets. In the era of precision medicine and accessible genome sequencing, we believe this will be a very useful data source for research and teaching purposes.

\section{Data availability}

Our subject agreed to release all of his genomic sequencing data for public use, which has been deposited into the European Genome-Phenome Archive (https://ega-archive. org; EGAS00001004648).

\section{Compliance with ethical standards}

Conflict of interest AJA reports research funding from consulting with Pfizer, Astellas, Merck, AstraZeneca, Janssen, Bayer, Dendreon, and research support (to Duke) from all above, plus BMS, Constellation, Gilead, Genentech/Roche. MR serves on the board of scientific advisors to NeoGenomics. He receives research support from Roche, Novartis, and Janssen. Weill Cornell Medicine has a patent on SPOP mutations in prostate cancer in the fields of diagnostics. MR is listed as a co-inventor. The other authors have declared no conflict of interest.

Publisher's note Springer Nature remains neutral with regard to jurisdictional claims in published maps and institutional affiliations.

Open Access This article is licensed under a Creative Commons Attribution 4.0 International License, which permits use, sharing, adaptation, distribution and reproduction in any medium or format, as long as you give appropriate credit to the original author(s) and the source, provide a link to the Creative Commons license, and indicate if changes were made. The images or other third party material in this article are included in the article's Creative Commons license, unless indicated otherwise in a credit line to the material. If material is not included in the article's Creative Commons license and your intended use is not permitted by statutory regulation or exceeds the permitted use, you will need to obtain permission directly from the copyright holder. To view a copy of this license, visit http://creativecommons.org/licenses/by/4.0/. 


\section{References}

1. Gundem G, Van Loo P, Kremeyer B, Alexandrov LB, Tubio JMC, Papaemmanuil E, et al. The evolutionary history of lethal metastatic prostate cancer. Nature. 2015;520:353-7.

2. Robinson D, Van Allen EM, Wu YM, Schultz N, Lonigro RJ, Mosquera JM, et al. Integrative clinical genomics of advanced prostate cancer. Cell. 2015;161:1215-28.

3. Liu W, Laitinen S, Khan S, Vihinen M, Kowalski J, Yu G, et al. Copy number analysis indicates monoclonal origin of lethal metastatic prostate cancer. Nat Med. 2009;15:559-65.

4. Cancer Genome Atlas Research Network. The molecular taxonomy of primary prostate cancer. Cell. 2015;163:1011-25.

5. Aryee MJ, Liu W, Engelmann JC, Nuhn P, Gurel M, Haffner MC, et al. DNA methylation alterations exhibit intraindividual stability and interindividual heterogeneity in prostate cancer metastases. Sci Transl Med. 2013;5:169ra10-ra10.

6. Zhao SG, Chang SL, Erho N, Yu M, Lehrer J, Alshalalfa M, et al. Associations of luminal and basal subtyping of prostate cancer with prognosis and response to androgen deprivation therapy. JAMA Oncol. 2017;3:1663-72.

7. Pritchard CC, Mateo J, Walsh MF, De Sarkar N, Abida W, Beltran $\mathrm{H}$, et al. Inherited DNA-repair gene mutations in men with metastatic prostate cancer. N. Engl J Med. 2016;375:443-53.

8. Le DT, Durham JN, Smith KN, Wang H, Bartlett BR, Aulakh LK, et al. Mismatch repair deficiency predicts response of solid tumors to PD-1 blockade. Science. 2017;357:409-13.

9. Mateo J, Carreira S, Sandhu S, Miranda S, Mossop H, PerezLopez R, et al. DNA-repair defects and olaparib in metastatic prostate cancer. N. Engl J Med. 2015;373:1697-708.

10. Wu Y-M, Cieślik M, Lonigro RJ, Vats P, Reimers MA, Cao X, et al. Inactivation of CDK12 delineates a distinct immunogenic class of advanced prostate cancer. Cell. 2018;173:1770-82. e14.

11. Antonarakis ES, Isaacsson Velho P, Fu W, Wang H, Agarwal N, Sacristan Santos V, et al. CDK12-altered prostate cancer: clinical features and therapeutic outcomes to standard systemic therapies, poly (ADP-ribose) polymerase inhibitors, and PD-1 inhibitors. JCO Precis Oncol. 2020;4:370-81.

12. Schweizer MT, Ha G, Gulati R, Brown LC, McKay RR, Dorff T, et al. CDK12-mutated prostate cancer: clinical outcomes with standard therapies and immune checkpoint blockade. JCO precision oncology. 2020;4:382-92.

13. Rennert H, Eng K, Zhang T, Tan A, Xiang J, Romanel A, et al. Development and validation of a whole-exome sequencing test for simultaneous detection of point mutations, indels and copynumber alterations for precision cancer care. NPJ Genom Med. 2016;1:16019.

14. Cibulskis K, Lawrence MS, Carter SL, Sivachenko A, Jaffe D, Sougnez C, et al. Sensitive detection of somatic point mutations in impure and heterogeneous cancer samples. Nat Biotechnol. 2013;31:213-9.

15. Saunders CT, Wong WS, Swamy S, Becq J, Murray LJ, Cheetham RK. Strelka: accurate somatic small-variant calling from sequenced tumor-normal sample pairs. Bioinformatics. 2012;28:1811-7.

16. Campbell PJ, Getz G, Stuart JM, Korbel JO, Stein LD. Pan-cancer analysis of whole genomes. bioRxiv. https://www.biorxiv.org/ content/10.1101/162784v1. 2017.

17. McKenna A, Hanna M, Banks E, Sivachenko A, Cibulskis K, Kernytsky A, et al. The Genome Analysis Toolkit: a MapReduce framework for analyzing next-generation DNA sequencing data. Genome Res. 2010;20:1297-303.

18. Siva N. 1000 Genomes Project Consortium. An integrated map of genetic variation from 1,092 human genomes. Nature. 2012;491:56.
19. Ewing CM, Ray AM, Lange EM, Zuhlke KA, Robbins CM, Tembe WD, et al. Germline mutations in HOXB13 and prostatecancer risk. N. Engl J Med. 2012;366:141-9.

20. Chang K-H, Li R, Kuri B, Lotan Y, Roehrborn CG, Liu J, et al. A gain-of-function mutation in DHT synthesis in castration-resistant prostate cancer. Cell. 2013;154:1074-84.

21. Agarwal N, Hahn AW, Gill DM, Farnham JM, Poole AI, CannonAlbright L. Independent validation of effect of HSD3B1 genotype on response to androgen-deprivation therapy in prostate cancer. JAMA Oncol. 2017;3:856-7.

22. Hearn JWD, AbuAli G, Reichard CA, Reddy CA, Magi-Galluzzi $\mathrm{C}$, Chang $\mathrm{KH}$, et al. HSD3B1 and resistance to androgendeprivation therapy in prostate cancer: a retrospective, multicohort study. Lancet Oncol. 2016;17:1435-44.

23. Li Z, Alyamani M, Li J, Rogacki K, Abazeed M, Upadhyay SK, et al. Redirecting abiraterone metabolism to fine-tune prostate cancer anti-androgen therapy. Nature. 2016;533:547.

24. Fu Y, Liu Z, Lou S, Bedford J, Mu XJ, Yip KY, et al. FunSeq2: a framework for prioritizing noncoding regulatory variants in cancer. Genome Biol. 2014;15:480.

25. McLaren W, Gil L, Hunt SE, Riat HS, Ritchie GR, Thormann A, et al. The ensembl variant effect predictor. Genome Biol. 2016; 17:122.

26. Li S, Crawford FW, Gerstein MB. SigLASSO: a LASSO approach jointly optimizing sampling likelihood and cancer mutation signatures. bioRxiv. https://www.biorxiv.org/content/10. 1101/366740v2

27. Forbes SA, Beare D, Gunasekaran P, Leung K, Bindal N, Boutselakis $\mathrm{H}$, et al. COSMIC: exploring the world's knowledge of somatic mutations in human cancer. Nucleic Acids Res. 2015;43: D805-11.

28. Alexandrov LB, Jones PH, Wedge DC, Sale JE, Campbell PJ, Nik-Zainal S, et al. Clock-like mutational processes in human somatic cells. Nat Genet. 2015;47:1402-7.

29. Consortium ITP-CAoWG. Pan-cancer analysis of whole genomes. Nature. 2020;578:82-93.

30. Mroz EA, Rocco JW. MATH, a novel measure of intratumor genetic heterogeneity, is high in poor-outcome classes of head and neck squamous cell carcinoma. Oral Oncol. 2013;49:211-5.

31. Armenia J, Wankowicz SA, Liu D, Gao J, Kundra R, Reznik E, et al. The long tail of oncogenic drivers in prostate cancer. Nat Genet. 2018;50:645.

32. Romanel A, Garritano S, Stringa B, Blattner M, Dalfovo D, Chakravarty D, et al. Inherited determinants of early recurrent somatic mutations in prostate cancer. Nat Commun. 2017;8:48.

33. Ernst J, Kellis M. ChromHMM: automating chromatin-state discovery and characterization. Nat Methods. 2012;9:215.

34. An J, Wang C, Deng Y, Yu L, Huang H. Destruction of fulllength androgen receptor by wild-type SPOP, but not prostatecancer-associated mutants. Cell Rep. 2014;6:657-69.

35. Shenoy TR, Boysen G, Wang MY, Xu QZ, Guo W, Koh FM, et al. CHD1 loss sensitizes prostate cancer to DNA damaging therapy by promoting error-prone double-strand break repair. Ann Oncol. 2017;28:1495-507.

36. Barbieri CE, Baca SC, Lawrence MS, Demichelis F, Blattner M, Theurillat J-P, et al. Exome sequencing identifies recurrent SPOP, FOXA1 and MED12 mutations in prostate cancer. Nat Genet. 2012;44:685.

37. Abida W, Cyrta J, Heller G, Prandi D, Armenia J, Coleman I, et al. Genomic correlates of clinical outcome in advanced prostate cancer. Proc Natl Acad Sci USA. 2019;116:11428-36.

38. Abida W, Armenia J, Gopalan A, Brennan R, Walsh M, Barron D, et al. Prospective genomic profiling of prostate cancer across disease states reveals germline and somatic alterations that may affect clinical decision making. JCO precision oncology. 2017;1:1-6. 
39. Zhang D, Wang H, Sun M, Yang J, Zhang W, Han S, et al. Speckle-type POZ protein, SPOP, is involved in the DNA damage response. Carcinogenesis. 2014;35:1691-7.

40. Boysen G, Barbieri CE, Prandi D, Blattner M, Chae S-S, Dahija A, et al. SPOP mutation leads to genomic instability in prostate cancer. Elife. 2015;4:e09207.

41. Swami U, Velho PI, Nussenzveig R, Chipman J, Santos VS, Erickson S, et al. Hahn AW. Association of SPOP mutations with outcomes in men with de novo metastatic castration-sensitive prostate cancer. European Urology. 2020;78:652-6.
42. Drilon A, Cappuzzo F, Ou S-HI, Camidge DR. Targeting MET in lung cancer: will expectations finally be MET? J Thorac Oncol. 2017;12:15-26.

43. Smith M, De Bono J, Sternberg C, Le Moulec S, Oudard S, De Giorgi U, et al. Phase III study of cabozantinib in previously treated metastatic castration-resistant prostate cancer: COMET-1. J Clin Oncol 2016;34:3005-13.

44. Miranda-Gonçalves V, Lameirinhas A, Henrique R, Jerónimo C. Metabolism and epigenetic interplay in cancer: regulation and putative therapeutic targets. Frontiers in genetics. 2018;9:427.

\section{Affiliations}

\section{Andrew J. Armstrong $\mathbb{D}^{1} \cdot$ Xiaotong $\mathrm{Li}^{2} \cdot$ Matthew Tucker $^{3} \cdot$ Shantao $\mathrm{Li}^{2} \cdot$ Xinmeng Jasmine $\mathrm{Mu}^{4}$. Kenneth Wha $\mathrm{Eng}^{5} \cdot$ Andrea Sboner $^{6} \cdot$ Mark Rubin $\mathbb{1}^{6,7,8} \cdot$ Mark Gerstein $\mathbb{1}^{2,9}$}

1 Duke Cancer Institute Center for Prostate and Urologic Cancer, Departments of Medicine, Surgery, Pharmacology and Cancer Biology, Duke Cancer Institute, Durham, NC, USA

2 Program in Computational Biology and Bioinformatics, Yale University, New Haven, CT, USA

3 Department of Medicine, Vanderbilt University Medical Center, Nashville, TN, USA

4 The Broad Institute of MIT \& Harvard, Cambridge, MA, USA

5 Department of Physiology and Biophysics, Englander Institute for Precision Medicine, HRH Prince Alwaleed Bin Talal Bin Abdulaziz Alsaud Institute for Computational Biomedicine, Weill Cornell Medical College, New York, NY, USA
6 Department of Pathology and Laboratory Medicine, Englander Institute for Precision Medicine, HRH Prince Alwaleed Bin Talal Bin Abdulaziz Alsaud Institute for Computational Biomedicine, Meyer Cancer Center, Weill Cornell Medical College, New York, NY, USA

7 Department for BioMedical Research, University of Bern and Inselspital, 3010 Bern, Switzerland

8 Bern Center for Precision Medicine, University of Bern and Inselspital, 3010 Bern, Switzerland

9 Department of Molecular Biophysics and Biochemistry, Department of Statistics and Data Science, Department of Computer Science, Yale University, New Haven, CT, USA 